\title{
Electrical conductivity, ionic conductivity, optical absorption, and gas separation properties of ionically conductive polymer membranes embedded with Si microwire arrays
}

\author{
Joshua M. Spurgeon, ${ }^{a}$ Michael G. Walter, ${ }^{a}$ Junfeng Zhou, ${ }^{b}$ Paul A. Kohl ${ }^{* b}$ and Nathan S. Lewis ${ }^{a}$ \\ Received 11th January 2011, Accepted 23rd February 2011 \\ DOI: 10.1039/c1ee01028j
}

\begin{abstract}
The optical absorption, ionic conductivity, electronic conductivity, and gas separation properties have been evaluated for flexible composite films of ionically conductive polymers that contain partially embedded arrays of ordered, crystalline, p-type Si microwires. The cation exchange ionomer Nafion, and a recently developed anion exchange ionomer, poly(arylene ether sulfone) that contains quaternary ammonium groups (QAPSF), produced composite microwire array/ionomer membrane films that were suitable for operation in acidic or alkaline media, respectively. The ionic conductivity of the Si wire array/ Nafion composite films in $2.0 \mathrm{M} \mathrm{H}_{2} \mathrm{SO}_{4}(\mathrm{aq})$ was $71 \mathrm{mS} \mathrm{cm}^{-1}$, and the conductivity of the Si wire array/ QAPSF composite films in $2.0 \mathrm{M} \mathrm{KOH}(\mathrm{aq})$ was $6.4 \mathrm{mS} \mathrm{cm}^{-1}$. Both values were comparable to the conductivities observed for films of these ionomers that did not contain embedded $\mathrm{Si}$ wire arrays. Two $\mathrm{Si}$ wire array/Nafion membranes were electrically connected in series, using a conducting polymer, to produce a trilayer, multifunctional membrane that exhibited an ionic conductivity in $2.0 \mathrm{M} \mathrm{H}_{2} \mathrm{SO}_{4}(\mathrm{aq})$ of $57 \mathrm{mS} \mathrm{cm}{ }^{-1}$ and an ohmic electrical contact, with an areal resistance of $\sim 0.30 \Omega \mathrm{cm}^{2}$, between the two physically separate embedded Si wire arrays. All of the wire array/ionomer composite membranes showed low rates of hydrogen crossover. Optical measurements indicated very low absorption $(<3 \%)$ in the ionexchange polymers but high light absorption (up to $80 \%$ ) by the wire arrays even at normal incidence, attesting to the suitability of such multifunctional membranes for application in solar fuels production.
\end{abstract}

a Beckman Institute and Kavli Nanoscience Institute, California Institute of Technology, Division of Chemistry and Chemical Engineering, 1200 E. California Blvd. $\mathrm{m} / \mathrm{c}$ 127-72, Pasadena, CA, 91125, USA. E-mail: nslewis@caltech.edu

${ }^{b}$ School of Chemical and Biomolecular Engineering, 311 Ferst Drive, N.W., Atlanta, GA, 30332, USA. E-mail: paul.kohl@chbe.gatech.edu

\section{Introduction}

An artificial photosynthetic assembly could be achieved, in principle, by combining in series two semiconductors that have appropriate energy levels to provide the necessary photovoltage $(>1.23 \mathrm{~V})$ to split water into $\mathrm{H}_{2}$ and $\mathrm{O}_{2}$, in conjunction with electrocatalysts to drive at low overpotentials the fuel-forming anodic and cathodic reactions. ${ }^{1-6}$ In one possible implementation of this approach (Fig. 1), a membrane would support and

\section{Broader context}

The widespread implementation of solar energy as a primary energy source will require the ability to overcome the diurnal variation of sunlight. The ideal solution would be to store solar energy in the form of chemical bonds, i.e., to convert sunlight into an energydense fuel. Significant progress has been made towards harnessing the sun to split water and make hydrogen fuel, but inherent physical limitations make it very difficult for a single material to efficiently and stably evolve hydrogen in sunlight. Combining two semiconductors in series circumvents some of these issues by providing a higher photovoltage while making more efficient use of the solar spectrum, similar to multijunction solar cells. Additionally, for a solar fuel generation device, the products must be separated to prevent recombination and minimize explosion hazards. The incorporation of the semiconductor photoanode and photocathode into a single membrane makes it possible to have a fully integrated, lightweight, flexible system to accomplish all of these processes without the need for external wires to effect electrical connections between components of the system. The work described herein demonstrates proof-of-concept test membranes that could form the basis for one such implementation of a solar fuel generation structure. 


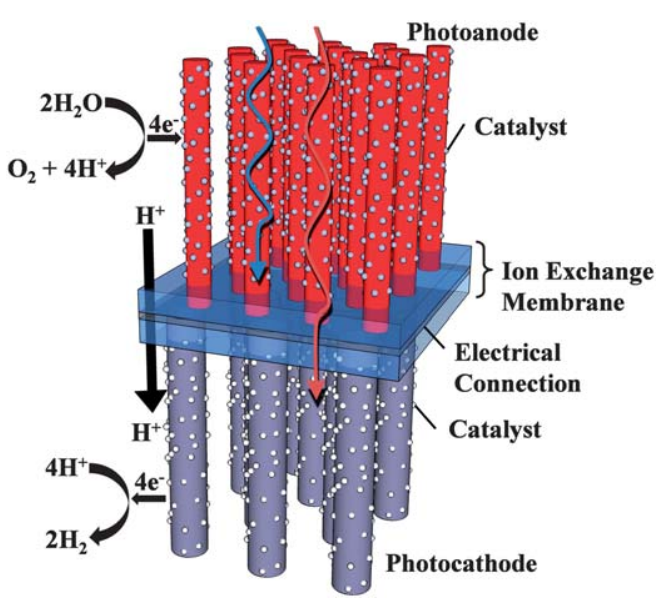

Fig. 1 Schematic of a proposed water-splitting device that would generate fuel from sunlight. The device uses two different semiconductors, a wider band gap photoanode material and a narrower band gap photocathode material, to produce the $>1.23 \mathrm{~V}$ necessary to electrolyze water. The anode material absorbs higher energy light, allowing lower energy light to be absorbed by the cathode. Catalysts distributed along the semiconductor surface facilitate the reactions at low overpotentials. The two semiconductors are electrically connected in a transparent membrane that is impermeable to $\mathrm{H}_{2}$ and $\mathrm{O}_{2}$ but allows ion transfer (presented as $\mathrm{H}^{+}$ in the schematic, but that could be $\mathrm{OH}^{-}$in a high $\mathrm{pH}$ environment). The semiconductors are radial junction arrays in order to utilize lower-purity materials, to distribute charge-carriers over a larger area so that the catalyst turnover requirement is lower, and to allow ion transfer across the membrane. $\mathrm{H}_{2}$ is collected on the cathode side and $\mathrm{O}_{2}$ is vented to the atmosphere from the anode side. The image is not to scale.

incorporate the two semiconductor materials, while maintaining an effective separation between the gaseous products and allowing ions to pass to neutralize the $\mathrm{pH}$ gradient. The collective incorporation of the two semiconductor materials and the requisite electrocatalysts into a single membrane would enable the formation of a fully integrated system that would not require any external electrical wiring connections to act as an artificial photosynthetic solar fuels generator. We describe the modular design and characterization of an ion exchange membrane that contains $\mathrm{Si}$ microwire arrays embedded in ionically conductive polymer separator films, which serves as an initial implementation of such an architecture.

In such a system, microstructuring the semiconductor absorber materials into high-aspect ratio wires would enable the use of materials that have low minority-carrier diffusion lengths, by providing a short path for carrier collection along the radial dimension of the wire. ${ }^{7}$ Accordingly, ordered arrays of semiconductor wires have shown promise in solar energy-conversion applications. ${ }^{8-10}$ Because of the enhanced surface area of a wire array relative to that of a planar geometry, the charge-carrier flux to the surface would be decreased, reducing the required turnover frequency at catalyst sites and potentially allowing the use of more abundant, less active electrocatalysts to effect the desired fuel-forming reactions at low overpotentials. Additionally, the space between wires provides a path for the conduction of ions across a membrane that separates the two semiconductor materials, completing the water-splitting reaction and preventing the buildup of a $\mathrm{pH}$ gradient. For the device to split water efficiently and continuously, the membrane must provide structural support for the wire arrays, act as an exchange medium for the ions produced and/or destroyed by the anodic and cathodic electrochemical reactions, separate the gaseous hydrogen and oxygen products, and enable an ohmic conduction path for electrons between the anode and cathode, while also providing sufficient optical transparency to ensure that light is effectively absorbed by both semiconductor assemblies (Fig. 1).

p-type $\mathrm{Si}$ is a prime candidate for the photocathode material because it is cathodically stable under illumination in acidic aqueous media ${ }^{11}$ and has been demonstrated, in conjunction with various metal catalysts, ${ }^{12}$ to evolve $\mathrm{H}_{2}(\mathrm{~g})$ from $\mathrm{H}_{2} \mathrm{O}$. The photoanode material will need to be a wider band gap semiconductor, most likely a metal oxide, that is stable in an oxidizing environment. ${ }^{5}$ In the present work, $\mathrm{p}$-Si was used on both sides of the multilayer membrane, to demonstrate the feasibility of fabricating such a system and of incorporating any similar semiconductor wire array into a suitable membrane structure, as well as to allow determination of the properties of an integrated multifunctional, multilayer ionomer/microwire array assembly.

Nafion, a perfluorosulfonic acid polytetrafluoroethylene copolymer, is commonly used as the membrane separator material in proton exchange membrane fuel cells. ${ }^{13,14}$ Accordingly, some solar hydrogen production studies have utilized composites of semiconductor nanoparticles and Nafion. ${ }^{15,16}$ One of the many challenges in the fabrication of the proposed dualsemiconductor water-splitting device is to successfully embed a wire array assembly into a robust, transparent, proton exchange film while simultaneously exposing the majority of the wire surface for the reaction and exposing the back end of the wires to allow for electrical connection to the other electrode. Wire arrays have successfully been transferred in this manner to thin films of polydimethylsiloxane (PDMS), ${ }^{17,18}$ but PDMS does not provide the ionic conductivity needed for a photosynthetic membrane material. Hence, in this work we have explored the fabrication and properties of single layer and multilayer Nafion/ $\mathrm{Si}$ wire array composites, to characterize this part of a membrane-bound artificial photosynthetic device.

Although Nafion exhibits good protonic conductivity in acidic media, Nafion does not function well at neutral $\mathrm{pH}$ or under alkaline conditions. The ability to operate the photoelectrolysis device at high $\mathrm{pH}$ could relax many of the device design constraints, by enabling the use of highly active, non-noble metal electrocatalysts in alkaline media, ${ }^{19-21}$ and by enabling the use of semiconductors that are not stable under acidic conditions. Several promising polymeric materials have recently been developed for application in an anionic fuel cell. ${ }^{22-25}$ For example, the anion exchange ionomer poly(arylene ether sulfone), functionalized with quaternary ammonium groups (QAPSF), is capable of exchanging hydroxide ions. ${ }^{25}$ This material has been used in our work to fabricate test $\mathrm{p}$-Si wire array/QAPSF membranes that can operate under alkaline conditions. Without adequate surface protection, Si will corrode in strong base, ${ }^{26}$ but the native oxide on the $\mathrm{Si}$ wires was sufficient to prevent significant corrosion during the time period needed to characterize the resulting membranes and to evaluate their performance for such applications. An effective surface protection scheme or the substitution of an alkaline-stable semiconductor wire array in place of Si should yield a membrane that is functional in an alkaline environment. 


\section{Experimental}

\section{A. Membrane fabrication}

$\mathrm{Si}$ wires were grown from a patterned $\mathrm{Cu}$ catalys $\mathrm{t}^{27}$ and were doped p-type, ${ }^{8}$ yielding wires that were $90 \pm 15 \mu \mathrm{m}$ long and $\sim 1.5$ to $1.7 \mu \mathrm{m}$ in diameter, in a square arrangement with a $7.0 \mu \mathrm{m}$ pitch. Solutions of Nafion in $N, N$-dimethylformamide (DMF) were prepared by addition of $1.2 \mathrm{~mL}$ of DMF to $3.0 \mathrm{~mL}$ of perfluorosulfonic acid-polytetrafluoroethylene (PTFE) copolymer (Nafion, $0.9 \mathrm{meq} \mathrm{g}^{-1}$ exchange capacity, $5 \% \mathrm{w} / \mathrm{w}$ in a water/ alcohol mixture, Alfa Aesar), followed by heating at $140{ }^{\circ} \mathrm{C}$ to evaporate the water/alcohol mixture until the solution volume was reduced to $\sim 1.2 \mathrm{~mL} .{ }^{28}$ This solution was spin-coated onto the wire arrays at $1000 \mathrm{rpm}$ for $30 \mathrm{~s}$, and the arrays were then heated at $140^{\circ} \mathrm{C}$ for $\sim 20 \mathrm{~min}$. This process was performed three times on each wire array, and produced a $\sim 50 \mu$ m thick Nafion film at the base of the wires. The Si wire array/Nafion composite membranes were then mechanically removed from the underlying $\mathrm{Si}(111)$ substrate using a razor blade. Membrane areas were typically $>3 \mathrm{~cm}^{2}$, to fully cover the glass flanges in the permeation cell that was used for the characterization experiments.

Dual Si wire array/Nafion membranes that were connected by an intervening layer of poly(3,4-ethylenedioxythiophene)poly(styrene sulfonate) (PEDOT-PSS) incorporated two such Si wire array/Nafion composites into a single film (Scheme 1). Each Si wire array/Nafion composite film was laid out on a thin sheet of flexible plastic, with the wire-base side facing upwards. Ethanol was dripped onto the Si wire array/Nafion membranes, causing the films to expand significantly. The resulting films were then carefully flattened against the plastic substrate. 2-3 drops of
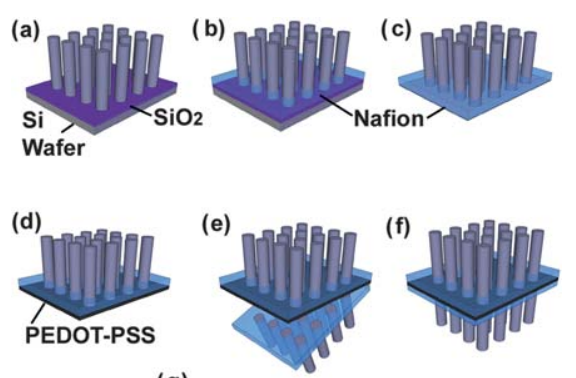

(g)

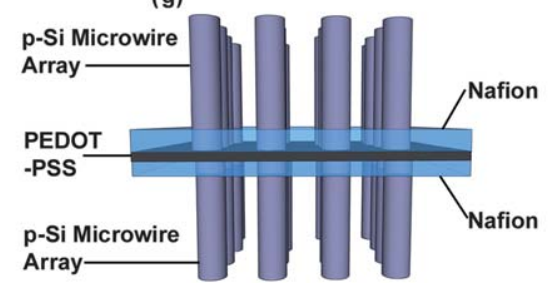

Scheme 1 Diagram of the fabrication process for a dual (Si wire array/ Nafion)/PEDOT-PSS composite membrane, illustrating the multicomponent structure. To make the multilayer membranes, (a) Si microwire arrays were grown on single crystal $\mathrm{Si}$ wafers that had been covered with a $\mathrm{SiO}_{2}$ surface template, (b) Nafion was cast from solution onto the bases of the $\mathrm{Si}$ wires, (c) the $\mathrm{Si}$ wire array/Nafion film was mechanically separated from the wafer, (d) electrically conductive PEDOT-PSS was applied to the back of the film, (e) a second Si wire array/Nafion film was pressed onto the other side of the PEDOT-PSS, and (f) the PEDOTPSS was allowed to dry to yield (g) a dual (Si wire array/Nafion)/ PEDOT-PSS membrane structure. The diagram is not to scale. a high conductivity dispersion of PEDOT-PSS, $2.2-2.6 \%$ in $\mathrm{H}_{2} \mathrm{O}$ (Aldrich), were then spin-cast at low rpm onto one of the membranes. The two $\mathrm{Si}$ wire array/Nafion films were then combined into a single membrane by pressing the two flexible plastic sheets together, with careful alignment of the films from one side to the other to prevent the incorporation of bubbles at the film-film interface. The dual (Si wire array/Nafion)/PEDOTPSS membrane was allowed to dry at room temperature and then peeled off of the plastic sheets.

Solution-cast Nafion membranes that did not contain embedded Si wires were prepared by drop-casting $0.60 \mathrm{~mL}$ of a Nafion solution in DMF (as prepared above) into a $1.7 \mathrm{~cm}$ diameter, open-ended glass tube that had been positioned vertically on a PDMS-coated glass slide. The solution was heated at $140{ }^{\circ} \mathrm{C}$ until it dried, yielding a Nafion film that was $50-70 \mu \mathrm{m}$ thick across the center. The film was peeled from the PDMS and cut from the bottom of the glass tube. Commercially prepared Nafion films were obtained by manually cutting samples from a commercially available fuel cell membrane electrode assembly (Nafion 115, $125 \mu \mathrm{m}$ thick, Clean Fuel Cell Energy).

Hydroxide exchange membranes were prepared using the anion exchange ionomer poly(arylene ether sulfone) that had been functionalized with quaternary ammonium groups (QAPSF). ${ }^{25} 0.050 \mathrm{~g}$ of QAPSF was dissolved in $0.50 \mathrm{~mL}$ of DMF, and the solution was spin-coated onto the wire arrays at $1000 \mathrm{rpm}$ for $30 \mathrm{~s}$. The films were then dried at room temperature for $>1 \mathrm{~h}$. This process was performed three times on each wire array, to create a $\sim 40 \mu \mathrm{m}$ thick QAPSF film at the base of the wires. The Si wire array/QAPSF composite membranes were then mechanically removed from the underlying Si substrate using a razor blade. QAPSF membranes that did not contain $\mathrm{Si}$ wires were prepared by casting films of the pure ionomer onto a glass slide. All of the QAPSF films were converted to the hydroxide form by soaking the membranes in $0.10 \mathrm{M} \mathrm{KOH}(\mathrm{aq})$ for $\sim 2 \mathrm{~h}$, with periodic rinsing in water, to exchange the $\mathrm{Cl}^{-}$ions for $\mathrm{OH}^{-}$ions.

\section{B. Membrane thickness measurements}

The thicknesses of films of commercially prepared Nafion, solution-cast Nafion, and solution-cast QAPSF were determined by measurement of the cross-sections of the membranes using an Olympus model BX51 optical microscope. Scanning electron microscope (SEM) images of cross-sections of the wire array/ ionomer films indicated that the thickness varied somewhat due to the tendency of the ionomer material to cling to the sides of the wires. The approximate thickness of the film was relatively constant across the membrane area. The average thickness of the wire array/ionomer composite films was estimated by determining the density of the Nafion or QAPSF (at $20^{\circ} \mathrm{C}$, relative humidity $\sim 60 \%$ ) from a solution-cast standard, precisely measuring the projected area of the wire array/ionomer composite film, weighing the film (at $20{ }^{\circ} \mathrm{C}$, relative humidity $\sim 60 \%$ ), and then using the density of the material to determine an average thickness while correcting for the presence of the $\mathrm{Si}$ wires (a small correction, $<15 \%$ of the mass). This average thickness was compared to the approximate value estimated from SEM cross-sectional images, and the values were in relatively close agreement (within $\sim 15 \%$ ) with each other. 


\section{Ionic conductivity measurements}

The ionic conductivity of the membranes was measured using a DC galvanodynamic method. ${ }^{29}$ To ensure full hydration, Nafion membranes were first immersed in $0.60 \mathrm{M} \mathrm{H}_{2} \mathrm{O}_{2}(\mathrm{aq})$ at $80{ }^{\circ} \mathrm{C}$ for $\sim 2 \mathrm{~h}$, followed by immersion in $0.50 \mathrm{M} \mathrm{H}_{2} \mathrm{SO}_{4}(\mathrm{aq})$ for $>48 \mathrm{~h}$. The membranes were sealed with gaskets between two glass flanges in a glass permeation cell. The cell was maintained at room temperature $\left(20{ }^{\circ} \mathrm{C}\right)$ and contained either $2.0 \mathrm{M}$ $\mathrm{H}_{2} \mathrm{SO}_{4}(\mathrm{aq})$ (when testing Nafion) or $2.0 \mathrm{M} \mathrm{KOH}(\mathrm{aq})$ (when testing QAPSF) on each side of the membrane. A Pt mesh electrode that had been cleaned with aqua regia was positioned in each compartment of the cell. A Luggin capillary that utilized a Pt wire, in either $2.0 \mathrm{M} \mathrm{H}_{2} \mathrm{SO}_{4}(\mathrm{aq})$ or $2.0 \mathrm{M} \mathrm{KOH}(\mathrm{aq})$ (to match the bulk solution), was then introduced into each cell compartment, with the tip of the capillary positioned $<2 \mathrm{~mm}$ from the membrane. Each capillary tip was attached to the inside of the glass flange with mounting wax, to maintain the position of the tip during loading and unloading of the membrane in the cell. A linear galvanodynamic current sweep from 0 to $400 \mathrm{~mA}$ $\mathrm{cm}^{-2}$ between the two Pt electrodes was performed using a model SI 1286 Schlumberger potentiostat, while the potential was recorded at each Luggin capillary. The current was swept at 1 $\mathrm{mA} \mathrm{s}{ }^{-1}$, starting from $0 \mathrm{~mA} \mathrm{~cm} \mathrm{~cm}^{-2}$ (at the rest potential of the membrane), to produce positive or negative membrane potential differences. Plots of the potential difference $v s$. current displayed ohmic behavior, allowing the cell resistance, $R_{\text {cell }}$, to be extracted from the slope of the plot. The electrolyte resistance, $R_{\text {elec }}$, was determined by measurement of the potential difference as a function of current in the absence of the membrane. The membrane resistance was then calculated using the relationship $R_{\text {mem }}=R_{\text {cell }}-R_{\text {elec }}$. The ionic conductivity of the membrane, $\sigma$, was calculated using $\sigma=L /\left(R_{\mathrm{mem}} A\right)$, where $A$ is the area of the membrane exposed to the galvanodynamic sweep and $L$ is the thickness of the membrane. ${ }^{29}$

\section{Hydrogen crossover measurements}

The rate of hydrogen crossover in the membranes was measured by modifying an electrochemical technique used for fuel cells that operate with feeds of gaseous reactants. ${ }^{30}$ The test membranes were sealed with gaskets between two glass flanges in a glass permeation cell that was maintained at room temperature $\left(20^{\circ} \mathrm{C}\right)$ and that contained 2.0 $\mathrm{M} \mathrm{H}_{2} \mathrm{SO}_{4}$ (aq) on each side of the membrane. Pt mesh electrodes that had been cleaned with aquaregia were then positioned within a few $\mathrm{mm}$ of each side of the membrane surface. Pure $\mathrm{H}_{2}(\mathrm{~g})$ and $\operatorname{Ar}(\mathrm{g})$ were bubbled into the cathode and anode compartments, respectively, at $0.3 \mathrm{~L} \mathrm{~min}^{-1}$, under strong stirring conditions. The cell was left at open circuit for $\sim 1 \mathrm{~h}$ to reach steady-state conditions. The potential of the anode (Ar saturated side) was then swept at $1 \mathrm{mV} \mathrm{s}^{-1}$ from the rest potential to $800 \mathrm{mV}$ against the cathode $\left(\mathrm{H}^{+} / \mathrm{H}_{2}\right)$, using a Model SI 1286 Schlumberger potentiostat. The $\mathrm{H}_{2}$ concentration was determined by measurement of the mass-transportlimited oxidation current density at the electrode. Before measurement of the hydrogen crossover concentration for each sample, a similar measurement was made in which pure hydrogen gas was bubbled into each compartment of the permeation cell. The stirring in the anode compartment was adjusted for each sample to achieve the same value for the masstransport-limited hydrogen oxidation current for a hydrogensaturated solution at $1 \mathrm{~atm}$. This approach ensured that similar mass transport conditions were present for each hydrogen crossover measurement. After normalization by the area of the membrane that was exposed to the solution, the resulting $\mathrm{H}_{2}$ concentration data were used to deduce a relative flux of $\mathrm{H}_{2}$ crossover through the membrane, by referencing the measured steady-state $\mathrm{H}_{2}$ concentration data across the membrane to the known hydrogen crossover flux, which corresponds to a hydrogen crossover current density of $0.22 \mathrm{~mA} \mathrm{~cm}^{-2}$ for liquidequilibrated commercial Nafion of this thickness at $20{ }^{\circ} \mathrm{C} .{ }^{31}$

\section{E. Measurements of the electrical connectivity between p-Si wire arrays}

The electrical connectivity between wire arrays in a dual (Si wire array/Nafion)/PEDOT-PSS composite film was measured by evaporating a film of a metal that formed an ohmic contact to p$\mathrm{Si}$ onto opposite sides of the composite membrane. First, to remove the native oxide from the wires, the dual ( $\mathrm{Si}$ wire array/ Nafion)/PEDOT-PSS membrane was immersed for $10 \mathrm{~s}$ in $6 \mathrm{M}$ $\mathrm{HF}(\mathrm{aq})$, rinsed thoroughly, and dried with $\mathrm{N}_{2}(\mathrm{~g})$. The membrane was then positioned under a shadow mask and $\sim 200 \mathrm{~nm}$ of $\mathrm{Au}$ was thermally evaporated onto the $\mathrm{p}-\mathrm{Si}$ wire array/Nafion surface. The membrane was flipped over and carefully positioned under the shadow mask again to align the metal pads, and another $\sim 200 \mathrm{~nm}$ of Au was evaporated. Ag print was then used to affix two separate coiled $\mathrm{Cu}$ wires to the Au-covered spots on each side of the membrane. The membrane was trimmed to a projected area of $0.30 \mathrm{~cm}^{2}$, with the entire area covered by Au. An SI Model 1286 Schlumberger potentiostat was used to linearly sweep the voltage between the two sides of the membrane. The current-voltage behavior of the PEDOT-PSS contact was tested as-deposited and also after immersion of the membrane for 6 days in $18 \mathrm{M} \Omega \mathrm{cm}$ resistivity $\mathrm{H}_{2} \mathrm{O}$ (while the membrane was still wet as well as after the membrane had dried).

\section{F. Optical absorption measurements}

The apparatus and technique used for optical absorption measurements has been described previously. ${ }^{32}$ An integrating sphere was used to collect transmission, $T_{\text {opt }}$, and reflection, $R_{\text {opt }}$, data separately for each sample. The absorption, $A_{\text {opt }}$, of the film was then determined by $A_{\text {opt }}=1-R_{\text {opt }}-T_{\text {opt }}$. A tunable, collimated light source was obtained by coupling a supercontinuum laser (Fianium) to a monochromator along with a chopper and lock-in amplifier. Data were collected in $2 \mathrm{~nm}$ increments.

\section{Results}

\section{A. Morphology and physical characteristics of the membranes}

As shown in Fig. 2a, uniform Si wire array/Nafion or Si wire array/ QAPSF composite films could be consistently fabricated over areas $>3 \mathrm{~cm}^{2}$. The size of the $\mathrm{Si}$ wire array/ionomer membranes was limited only by the diameter of the reactor tube used to grow the wire arrays. Electrically connected dual Si wire array/Nafion membranes were fabricated by sealing two separate wire array/ Nafion membranes together using the conducting polymer 


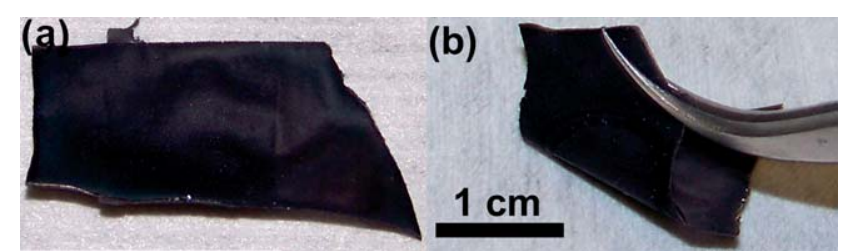

Fig. 2 Optical images of a multilayer Si wire array/Nafion composite membrane with an intervening layer of PEDOT-PSS. (a) Si wire array/ Nafion films were regularly fabricated with areas $>3 \mathrm{~cm}^{2}$. PEDOT-PSS was used to adhere two $\mathrm{Si}$ wire array/Nafion films together into (b) a mechanically robust, flexible membrane. The $1 \mathrm{~cm}$ scale bar applies to both images.

PEDOT-PSS. The resulting dual (Si wire array/Nafion)/PEDOTPSS membranes were highly flexible (Fig. 2b), and could be rolled and unrolled repeatedly without the two films separating or without the observable loss of Si wires. The SEM images presented
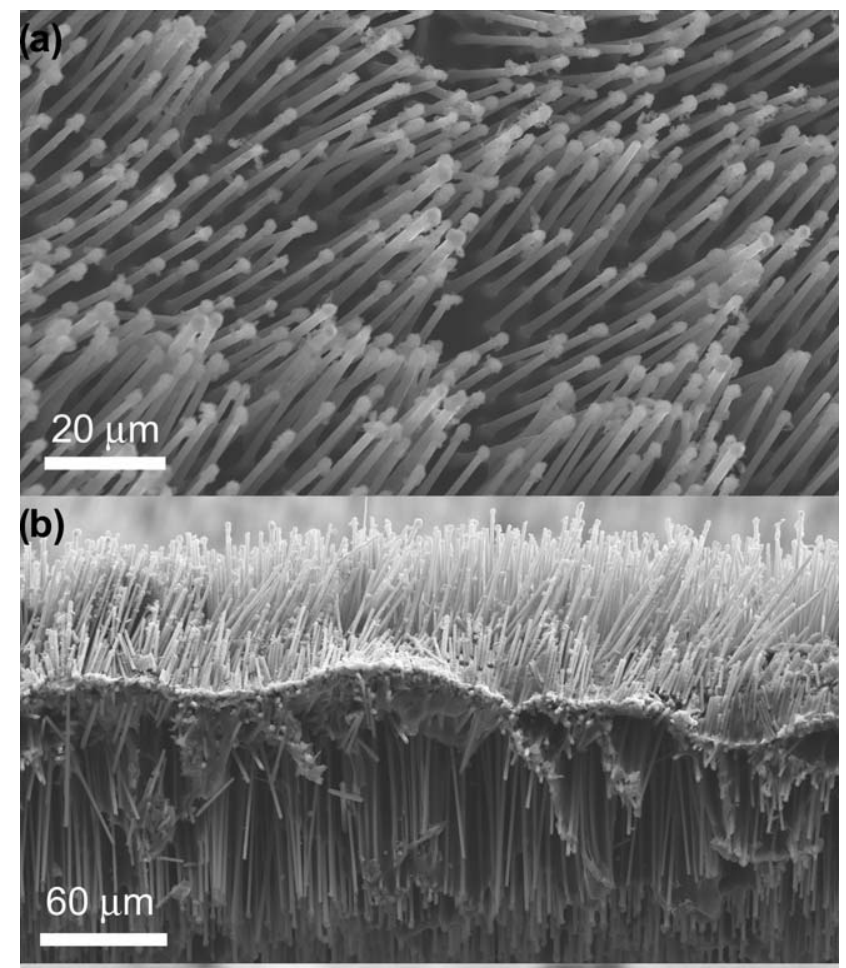

(c)

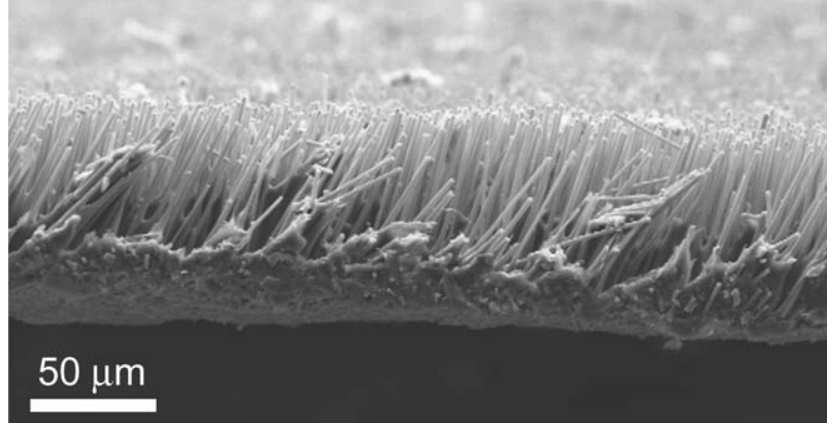

Fig. 3 SEM images of the (a) top-down view and (b) cross-sectional view of a dual Si wire array/Nafion composite membrane with an intervening layer of PEDOT-PSS, and the (c) cross-sectional view of a Si wire array/ QAPSF membrane. in Fig. 3 display the characteristic morphology that was observed for the $\mathrm{Si}$ wire arrays that had been embedded in the ionomer films. Both Nafion and QAPSF conformally coated the wires near the base of the array, making intimate contact with the Si without observable gaps between the wires and the ionomer.

\section{B. Ionic conductivity of the membranes}

To assess the ion-exchange properties of these membranes, the ionic conductivity of the films was determined using a fourelectrode DC technique. ${ }^{29}$ Table 1 shows the observed conductivity values. Measurements on pure samples of commercial Nafion 115, solution-cast Nafion, and solution-cast QAPSF films are also included for reference.

\section{Hydrogen crossover properties of the membranes}

In this work, an electrochemical technique used in fuel cell studies was adapted to determine a steady-state hydrogen crossover rate in an aqueous environment (see Experimental). ${ }^{30}$ Fig. 4 shows the observed hydrogen oxidation current densities for each type of membrane, and Table 1 shows the resulting hydrogen crossover current densities, as determined relative to a commercial Nafion standard. The hydrogen crossover current density of a thick plastic film with a single pinhole $\left(<0.5 \mathrm{~mm}^{2}\right)$ was $23 \mathrm{~mA} \mathrm{~cm}{ }^{-2}$.

\section{Electrical connectivity between p-Si wire arrays}

The electrical resistance between the two wire arrays was investigated by contacting the exposed wires on either side of the same area of a dual (Si wire array/Nafion)/PEDOT-PSS membrane (see Experimental). Fig. 5 shows the current-voltage behavior that resulted from a linear sweep of the voltage between each side of the membrane. The membrane was tested as fabricated and after soaking for 6 days in $\mathrm{H}_{2} \mathrm{O}$. The PEDOT-PSS film clearly produced an ohmic contact between the two Si wire array layers across the current range of interest. After immersion in water for 6 days, the areal resistance increased from 0.30 to $0.40 \Omega \mathrm{cm}^{2}$. After drying under ambient conditions, the resistance returned to $\sim 0.31 \Omega \mathrm{cm}^{2}$.

\section{E. Optical absorption properties of the membranes}

An integrating sphere setup was used to measure the absorption of films obtained by various permutations of the membrane components. ${ }^{32}$ The reported absorption is not the absorbance, but is the fraction of incident light that was absorbed by the samples. Fig. 6 displays the observed absorption at normal incidence across the visible region of the solar spectrum. Si wire array/ionomer composite membranes displayed absorption values as high as $\sim 0.80$ at wavelengths $<500 \mathrm{~nm}$, with the absorption tailing off toward the energy of the indirect band gap of $\mathrm{Si}$. The dual (Si wire array/Nafion)/PEDOT-PSS membrane displayed the highest absorption, reaching $\sim 0.90$ to 0.95 across most of the spectrum.

Fig. 7 shows the absorption vs. wavelength vs. angle of incidence for three types of Nafion membranes that contained $\mathrm{p}-\mathrm{Si}$ wire arrays. In each case, the absorption was only weakly affected by the angle of incidence. The $\mathrm{Si}$ wire array/Nafion composite absorption increased from a peak of $\sim 0.80$ at normal incidence to as high as $\sim 0.84$ at $\sim 40^{\circ}$. For the dual (Si wire array/ 
Table 1 Membrane characterization data

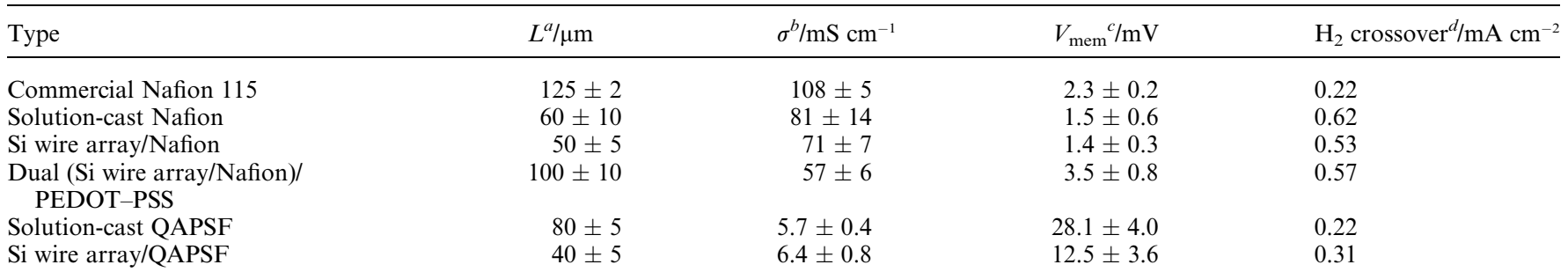

${ }^{a}$ Thicknesses do not include the length of $\mathrm{Si}$ wire exposed from the ionomer. ${ }^{b}$ Ionic conductivity measured at $20{ }^{\circ} \mathrm{C}$, in $2.0 \mathrm{M} \mathrm{H}_{2} \mathrm{SO}_{4}$ for $\mathrm{Nafion}$

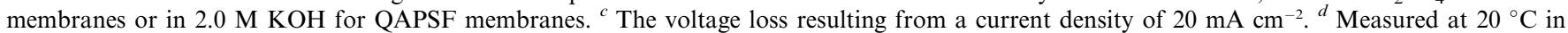
$2.0 \mathrm{M} \mathrm{H}_{2} \mathrm{SO}_{4}$, normalized to the commercial Nafion value, and multiplied by $0.22 \mathrm{~mA} \mathrm{~cm}{ }^{-2}$, the hydrogen crossover current density for commercial Nafion under these conditions. ${ }^{31}$

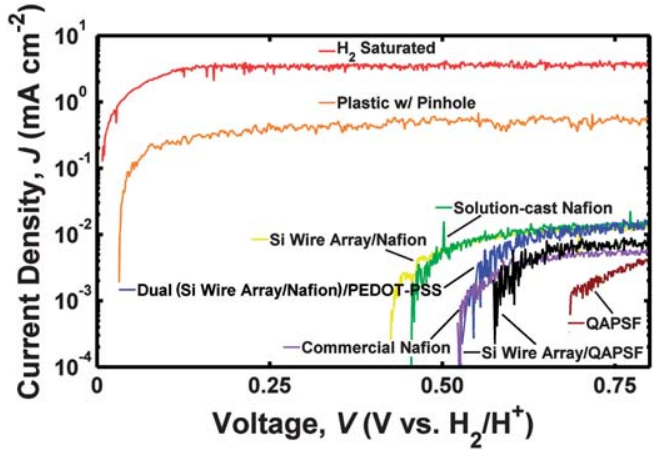

Fig. 4 Hydrogen oxidation current density, used to determine the hydrogen concentration, from linear sweep voltammograms for a $\mathrm{H}_{2} / \mathrm{Ar}$ saturated $\mathrm{H}_{2} \mathrm{SO}_{4}$ cell for each type of membrane, as well as for a plastic film with a pinhole. The voltammogram for a $\mathrm{H}_{2} / \mathrm{H}_{2}$ saturated $\mathrm{H}_{2} \mathrm{SO}_{4}$ cell is included for reference. The current density is plotted on a logarithmic scale.

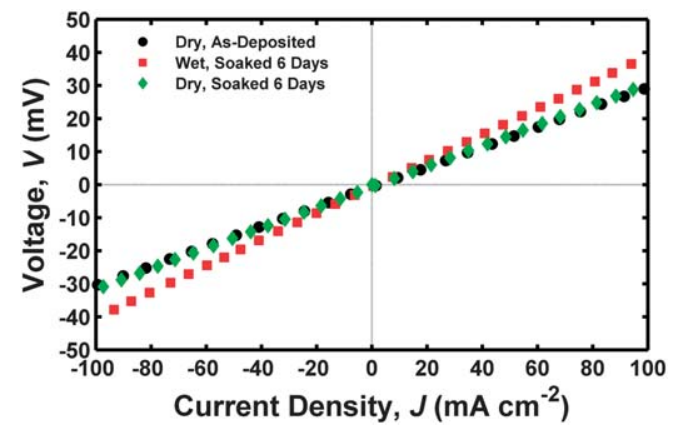

Fig. 5 Plot of voltage $v s$. current density $(V-J)$ for a dual Si wire array/ Nafion composite with an intervening layer of PEDOT-PSS that had been electrically contacted with $\mathrm{Au}$ on opposite sides of the membrane. The electrical contact was tested as-deposited and was then subsequently tested after the membrane had been soaked in water for 6 days (while the membrane was still wet and again after it had dried).

Nafion)/PEDOT-PSS membrane, the peak absorption increased from $\sim 0.96$ at normal incidence to $\sim 0.98$ at $\sim 40^{\circ}$.

\section{Discussion}

\section{A. Morphology and physical characteristics of the membranes}

To produce mechanically strong films, commercially available Nafion in a water/alcohol mixture was transferred to DMF for

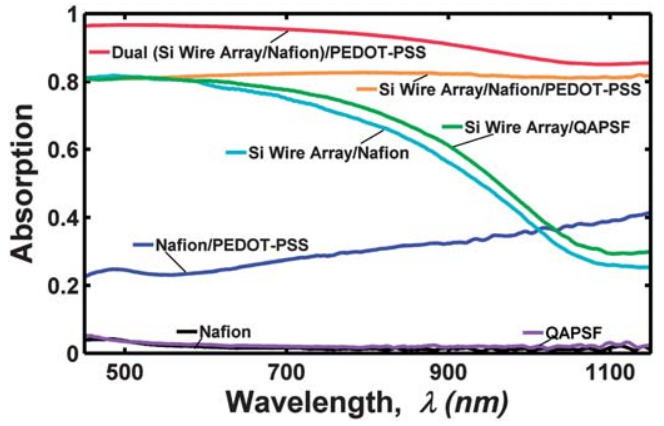

Fig. 6 Absorption vs. wavelength of light at normal incidence for each type of membrane.

solution casting, and the resulting films were cured at $140{ }^{\circ} \mathrm{C}$. The resulting large-area membranes were mechanically robust despite their thinness, did not dissolve in water, and were highly flexible (Fig. 2). Solution-cast Nafion is known to have poor mechanical quality, and furthermore Nafion partially dissolves in water, unless the Nafion is cast at elevated temperatures from suitable organic solvents. This latter process, used herein, improved the mechanical properties of the resulting Nafion films by allowing the Nafion to crystallize and produce ionic clusters that facilitated the exchange of protons. ${ }^{28}$ The flexibility provided by such membranes could allow the development of an integrated photoelectrolysis system that could be rolled out to cover larger areas. The ionomer films were thinner than the length of the $\mathrm{Si}$ microwires, allowing much of the $\mathrm{Si}$ wire surface area to be exposed from the membrane (Fig. 3). This ability to controllably expose the semiconductor wires from the membrane may be important to maximize the active surface area of catalyst that would decorate the semiconductor surface in a full photoelectrolysis device.

\section{B. Ionic conductivity of the membranes}

Each of the Nafion membranes showed a relatively high rate of exchange of protons. The values of $\sigma$ observed in this work for commercial Nafion films agreed with values that have been reported previously using this measurement technique. ${ }^{29}$ As expected, solution-cast Nafion displayed slightly lower ionic conductivity than commercial Nafion, and the conductivity of 


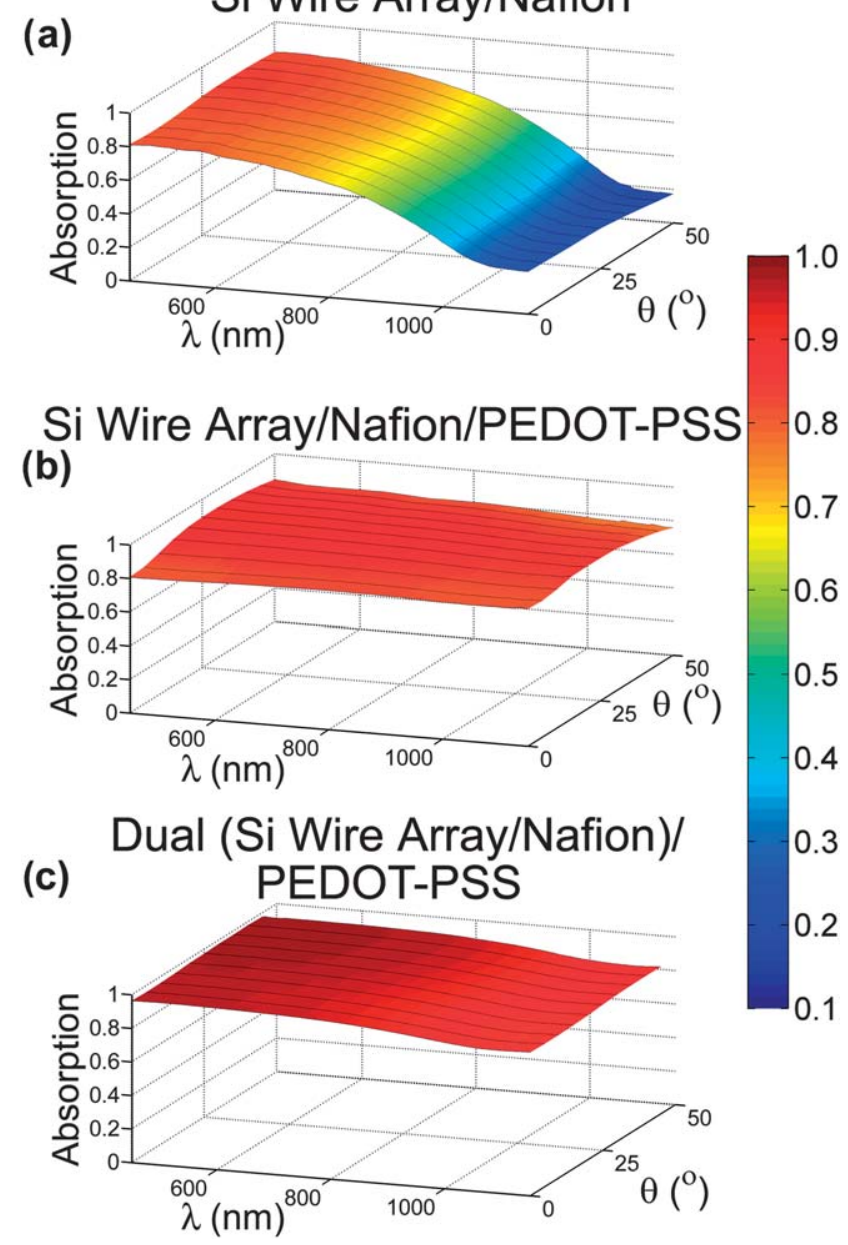

Fig. 7 Three-dimensional colorspace maps of absorption $v$ s. wavelength ( $\lambda$ ) vs. angle of incidence $(\theta)$. Absorption profile for (a) a Si wire array/ Nafion membrane, (b) a Si wire array/Nafion/PEDOT-PSS membrane, and (c) a dual Si wire array/Nafion membrane with an intervening layer of PEDOT-PSS.

the Si wire array/Nafion composite was comparable to that of pure solution-cast Nafion.

The addition of a thin PEDOT-PSS layer to adhere the two films together in the dual (Si wire array/Nafion)/PEDOT-PSS composite membrane only marginally reduced the ionic conductivity of the resulting film relative to that of a Si wire array/Nafion composite membrane. The minimal decrease in ionic conductivity is one advantage to the use of PEDOT-PSS to make an electrical connection to the $\mathrm{Si}$ wire arrays, because PEDOT-PSS has been demonstrated to have a reasonable ionic conductivity as well as a high electrical conductivity. ${ }^{33}$

The QAPSF-based membranes displayed significantly lower ionic conductivities than the Nafion films. Although hydroxide exchange materials with higher ionic conductivities have been reported, ${ }^{22-25}$ the QAPSF formulation was selected based on a combination of its mechanical robustness and flexibility as well as its low optical absorption. The mechanical properties of QAPSF allowed for the incorporation of Si wire arrays, while the high optical transparency of the QAPSF minimized the parasitic light absorption in the assembled membrane structures.
The ionic conductivity requirements of a photoelectrolysis membrane are much less stringent than those for a membrane used in a conventional electrolyzer or fuel cell. Under 1 Sun at the Earth's surface, a dual-semiconductor photoelectrolysis cell operating efficiently might optimistically produce current densities of $\sim 20 \mathrm{~mA} \mathrm{~cm}{ }^{-2}$ of projected area., ${ }^{2,3}$ The corresponding voltage loss, $V_{\text {mem }}$, associated with the ohmic resistance of the membrane can be determined by $V_{\text {mem }}=J R_{\text {mem }} A=J L / \sigma$, where $J$ is the current density of ions passing across the membrane. Under these conditions, the dual (Si wire array/Nafion)/PEDOT-PSS membrane would incur a voltage drop of only $3.5 \mathrm{mV}$ to exchange protons from the anode to the cathode in $2.0 \mathrm{M} \mathrm{H}_{2} \mathrm{SO}_{4}(\mathrm{aq})$ (Table 1). Even the $\mathrm{Si}$ wire array/QAPSF membrane, with a 10 times lower ionic conductivity than the $\mathrm{Si}$ wire array/Nafion analogue, would lose only $12.5 \mathrm{mV}$ to exchange hydroxide ions in $2.0 \mathrm{M}$ $\mathrm{KOH}(\mathrm{aq})$. The voltage loss from a dual (Si wire array/QAPSF)/ PEDOT-PSS membrane could thus be expected to be $\sim 30$ to $40 \mathrm{mV}$. Although this loss could likely be reduced with further optimization of the anion exchange ionomer, the lower overpotential that would result from the use of a non-noble metal catalyst at high $\mathrm{pH}$ rather than at low $\mathrm{pH}$ may compensate for this loss. Regardless, these voltages are small relative to the thermodynamically required $1.23 \mathrm{~V}$, and any additional catalytic overpotentials, that are necessary to split water.

\section{Hydrogen crossover properties of the membranes}

To act as an effective membrane, the separator material needs to be highly impermeable to the gaseous products to ensure efficient separation and to prevent $\mathrm{H}_{2}(\mathrm{~g})$ and $\mathrm{O}_{2}(\mathrm{~g})$ from recombining to form water. As the smaller, more diffusive species, $\mathrm{H}_{2}(\mathrm{~g})$ is generally more permeating, and thus, the impermeability of a membrane is usually evaluated by measurement of the rate of hydrogen crossover in the membrane separator of a fuel cell or electrolyzer. The electrochemical technique used in this work yielded the relative hydrogen crossover flux of the membranes; however, because the observed $\mathrm{H}_{2}$ concentration is mass-transport-dependent, the data do not provide an absolute value for the hydrogen crossover flux. The actual $\mathrm{H}_{2}(\mathrm{~g})$ crossover flux was estimated by normalizing the observed $\mathrm{H}_{2}$ concentration by the value observed for the commercial Nafion membrane, and scaling the data relative to the hydrogen crossover current density determined for this sample using the known properties of Nafion. ${ }^{31}$ The hydrogen crossover rate of a thick plastic film with a single pinhole $\left(<0.5 \mathrm{~mm}^{2}\right)$ shows the response that could be expected if the $\mathrm{Si}$ wire array/ionomer composite films contained multiple small perforations or gaps between the membrane and the semiconductor wires (Fig. 4). Instead, the hydrogen crossover rate of $\mathrm{Si}$ wire array/Nafion composite films was comparable to the value measured for commercial Nafion, indicating that the membrane maintained a high level of impermeability despite the $\mathrm{Si}$ wires embedded in the ionomer. The hydrogen crossover rate observed for Si wire array/QAPSF membranes was even lower than the crossover rate observed for the Si wire array/Nafion membranes. The open-circuit potential in each case, as described by the Nernst equation, is logarithmically dependent on the ratio of the concentration of $\mathrm{H}_{2}(\mathrm{~g})$ in the anode compartment relative to the $\mathrm{H}_{2}(\mathrm{~g})$ concentration in the cathode compartment. ${ }^{30}$ Thus, with increasing amounts of hydrogen crossover, the open-circuit 
potential vs. the cathode $\left(\mathrm{H}_{2} / \mathrm{H}^{+}\right)$generally decreased, and this trend agreed reasonably well with the observed limiting anodic current densities.

As an additional qualitative assurance that the membranes could exchange ions without significant gas crossover, membranes were tested in the permeation cell with stirred $\mathrm{pH} 6$ water on one side and either $\mathrm{pH} 1$ (for Nafion, using $\mathrm{H}_{2} \mathrm{SO}_{4}$ ) or $\mathrm{pH} 11$ (for QAPSF, using $\mathrm{KOH}$ ) water with blue dye on the other side. The $\mathrm{pH}$ of the clear, initially neutral side was monitored over time. In all cases, the $\mathrm{pH}$ changed significantly (decreasing for Nafion and increasing for QAPSF), but no dye crossover was observed for $>5$ $\mathrm{h}$. In contrast, for a film in which a small pinhole had been deliberately introduced, dye crossover was observable within minutes.

\section{Electrical connectivity between $\mathrm{p}-\mathrm{Si}$ wire arrays}

Unlike the membrane in a standard fuel cell or electrolyzer, the membrane for this particular implementation of a photoelectrolysis device must also electrically connect the anode to the cathode. This connection is a requirement for the series combination of the two semiconductor photovoltages needed to generate fuel. In the test membrane reported herein, the two p-Si wire arrays were electrically connected to each other by contacting the bases of the wires of each array to either side of a thin PEDOT-PSS layer. PEDOT-PSS is relatively conductive, and makes ohmic contact to p-type Si at low applied bias (Fig. 5). The observed increase in resistance in the presence of water is likely due to the resulting swelling of the membrane, which increases the area and lengthens the path for electrons across the conducting polymer. Upon drying, however, the resistance returned to approximately the same value as that measured before soaking. The PEDOT-PSS contact therefore did not appear to degrade significantly in liquid water. In addition, solution-cast PEDOT-PSS films adhered well to both dry and wet Nafion membranes, with no observed delamination of the conductive polymer. Although PEDOT-PSS is likely to swell when exposed to water, it is unlikely to become redispersed, as evidenced by the stability of free-standing cast films in contact with water.

For a photoelectrolysis device that is sustaining a current density of $20 \mathrm{~mA} \mathrm{~cm}^{-2}$ in water, the observed resistance of the PEDOT-PSS wire contact corresponds to an ohmic voltage loss of $\sim 8 \mathrm{mV}$. This value is an upper estimate of the ohmic resistance loss because no attempt was made to correct the measurement for the contact resistance to the outside of the membrane. In a finalized device that would utilize two different semiconductors, a modified approach will likely be needed to provide an ohmic contact to both electrode materials. Fully ohmic behavior in such a case could be achieved by combining two separate conducting polymers, one that makes ohmic contact to each electrode, or alternatively, by electrolessly depositing a very thin layer of an appropriate contact metal on the bases of the wires of each electrode before adhering the two films together with PEDOT-PSS.

\section{E. Optical absorption properties of the membranes}

A final criterion for the membrane in this dual semiconductor photoelectrolysis application is that the parasitic light absorption must be kept to a minimum. Any light absorbed by the polymer will not generate charge-carriers for the production of fuel. The optical absorption data of the various membrane structures in Fig. 6 can be analyzed to determine which components are absorbing the most light in each part of the spectrum. Solution-cast forms of both Nafion and QAPSF appear optically transparent at this thickness, with a reflection of $\sim 0.10$ and $\sim 0.15$, respectively, and a transmission of $\sim 0.88$ and $\sim 0.83$, respectively, across most of the visible spectrum at normal incidence. Despite the transparency of the ion-exchange polymer, $\mathrm{Si}$ wire array/Nafion or QAPSF composite membranes absorbed strongly (up to $\sim 0.80$ ), especially considering that the packing fraction of this pattern of Si wires is only $\sim 4 \%$. These absorption values are significantly higher than the absorption reported previously for this pattern of $\mathrm{Si}$ wire arrays at normal incidence without the presence of additional light-trapping features. ${ }^{32}$ While the absorption increased with angle of incidence (Fig. 7), the effect of angle was also less significant than reported previously. ${ }^{32}$ The absorption in this case is higher, and the effect of the angle of incidence is weaker, because much of the wire array is exposed from the polymer matrix. As a result, the wires are less vertically aligned, permitting less light to pass in between the wires. Additionally, a portion of the light that did pass between the wires reflected off the polymer surface and was largely redirected into the wires. The addition of a second, similar wire array connected by a conducting polymer resulted in very high absorption ( $\sim 0.90$ to 0.95$)$ across most of the spectrum. For this dual (Si wire array/Nafion)/PEDOT-PSS membrane, the absorption was nearly independent of the angle of incidence (Fig. 7). Light transmission through this composite film was nearly zero at all angles. This level of absorption is encouraging for the prospects of this solar fuel generation device design, in which it may be difficult to incorporate light-trapping effects such as an antireflection coating. For two different semiconductors, these types of optical absorption measurements will be a critical metric to adjust the length and spacing of the wires in order to maximize the light absorption and to match the current of the photoanode to that of the photocathode.

The PEDOT-PSS that was used to provide the electrical connection between the wire arrays is not as transparent as the ion-exchange materials. The addition of a relatively thin layer of PEDOT-PSS (estimated by SEM to be $<700 \mathrm{~nm}$ ) to Nafion or to a Si wire array/Nafion composite film increased the absorbance of the resulting multilayer membrane, particularly in the nearinfrared portion of the spectrum (Fig. 6 and 7). The relatively high absorption coefficient of PEDOT-PSS in the near-infrared is disadvantageous because this light will need to pass through the electrically conductive layer of the membrane to reach the narrower band gap semiconductor material in the full device (Fig. 1). This issue could be alleviated by thinning the PEDOTPSS layer or by electrochemically reducing the conducting polymer, the latter of which has been observed to lower the absorbance in the near-infrared. ${ }^{34}$ Either approach, however, would likely affect the electrical conductivity of this layer. Hence, further research should focus on the development of highly conductive, minimally absorbing layers for use in the connection of the two semiconductor arrays, or methods to self-align and connect the wires of two different, suitably light absorbing semiconductor materials to obviate entirely the need for the electrically conductive layer in the membrane. 


\section{Conclusions}

Semiconductor microwire arrays have been embedded in ionexchange membranes that are well-suited for operation at either low or high $\mathrm{pH}$. The resulting composite membranes were mechanically stable, flexible films that displayed low hydrogen crossover, despite the array of wires bridging across and protruding from the ionomer layer. The voltage loss that is likely to arise from resistance to ionic transport in these membranes is relatively small (from $\sim 4$ to $40 \mathrm{mV}$ ), and probably acceptable for a first-generation photoelectrolysis system. Two wire array/ionomer composite films were adhered together and electrically connected to each other using PEDOT-PSS without adversely affecting the ionic conductivity of the membrane. Very little light was lost by absorption $\left(A_{\mathrm{opt}}<0.03\right.$ across most of the visible spectrum) due to the ion-exchange polymers. The PEDOT-PSS connecting layer, however, did absorb a modest amount of light (up to $A_{\text {opt }} \sim 0.4$ at $1100 \mathrm{~nm}$ ). Modification of this layer to minimize parasitic light absorption and electrical resistance will be important in producing an optimally efficient device. Layer-by-layer techniques that can uniformly deposit extremely thin films of ionically and/or electrically conductive polymers are a promising route for the improvement of this connecting layer. ${ }^{35,36}$ This membrane design allows a modular approach, in which it should be possible to improve the various elements of the photoelectrolysis device relatively independently of each other. If two desirable photoanode and photocathode materials would ideally operate in different $\mathrm{pH}$ environments, this modularity may enable the electrodes to be combined in a hybrid Nafion/ QAPSF membrane, comparable to the hybrid membranes that have been demonstrated recently for use in fuel cells. ${ }^{37,38}$ Ultimately, dual semiconductor wire array/ionomer composites can be envisioned as photoelectrolysis membranes in an assembly that enables the energy-dense storage of solar energy directly as chemical fuel.

\section{Acknowledgements}

This work was supported by the Department of Energy, Office of Basic Energy Sciences, DE-FG02-07ER46405 and by DARPA contract \#W911NF-09-2-0011. We acknowledge use of facilities supported by the Caltech Center for Science and Engineering of Materials, an NSF MRSEC, and the Caltech Center for Sustainable Energy Research. The financial support (J.Z. and P.K.) from the Army Research Laboratory, contract LCHS22067 is gratefully acknowledged. M.G.W. acknowledges the financial support from an NSF-ACCF postdoctoral fellowship (CHE-0937048).

\section{References}

1 E. L. Johnson, IEEE Int. Electron Devices Meet., 1981, 27, 2-5.

2 O. Khaselev and J. A. Turner, Science, 1998, 280, 425-427.

3 S. Licht, B. Wang, S. Mukerji, T. Soga, M. Umeno and H. Tributsch, J. Phys. Chem. B, 2000, 104, 8920-8924.
4 S. Licht, J. Phys. Chem. B, 2001, 105, 6281-6294.

5 B. D. Alexander, P. J. Kulesza, L. Rutkowska, R. Solarska and J. Augustynski, J. Mater. Chem., 2008, 18, 2298-2303.

6 M. G. Walter, E. L. Warren, J. R. McKone, S. W. Boettcher, Q. X. Mi, E. A. Santori and N. S. Lewis, Chem. Rev., 2010, 110, 6446-6473.

7 B. M. Kayes, H. A. Atwater and N. S. Lewis, J. Appl. Phys., 2005, 97, 114302.

8 S. W. Boettcher, J. M. Spurgeon, M. C. Putnam, E. L. Warren, D. B. Turner-Evans, M. D. Kelzenberg, J. R. Maiolo, H. A. Atwater and N. S. Lewis, Science, 2010, 327, 185-187.

9 M. C. Putnam, S. W. Boettcher, M. D. Kelzenberg, D. B. TurnerEvans, J. M. Spurgeon, E. L. Warren, R. M. Briggs, N. S. Lewis and H. A. Atwater, Energy Environ. Sci., 2010, 3, 1037-1041.

10 E. C. Garnett and P. D. Yang, J. Am. Chem. Soc., 2008, 130, 9224-9225.

11 A. Heller, H. J. Lewerenz and B. Miller, J. Am. Chem. Soc., 1981, 103, 200-201.

12 M. Szklarczyk and J. O. M. Bockris, J. Phys. Chem., 1984, 88, 18081815.

13 J. Larminie and A. Dicks, Fuel Cell Systems Explained, John Wiley \& Sons, West Sussex, England, 2nd edn, 2003.

14 C. Heitner-Wirguin, J. Membr. Sci., 1996, 120, 1-33.

15 A. W. H. Mau, C. B. Huang, N. Kakuta, A. J. Bard, A. Campion, M. A. Fox, J. M. White and S. E. Webber, J. Am. Chem. Soc., 1984, 106, 6537-6542.

16 H. Park and W. Choi, Langmuir, 2006, 22, 2906-2911.

17 K. E. Plass, M. A. Filler, J. M. Spurgeon, B. M. Kayes, S. Maldonado, B. S. Brunschwig, H. A. Atwater and N. S. Lewis, Adv. Mater., 2009, 21, 325-328.

18 J. M. Spurgeon, S. W. Boettcher, M. D. Kelzenberg, B. S. Brunschwig, H. A. Atwater and N. S. Lewis, Adv. Mater., 2010, 22, 3277-3281.

19 S. Trasatti, in Advances in Electrochemical Science and Engineering vol. 2, VCH, 1992, pp. 1-85.

20 E. L. Miller and R. E. Rocheleau, J. Electrochem. Soc., 1997, 144, 3072-3077.

21 R. E. Rocheleau, E. L. Miller and A. Misra, Energy Fuels, 1998, 12, 3-10.

22 S. Gu, R. Cai, T. Luo, Z. W. Chen, M. W. Sun, Y. Liu, G. H. He and Y. S. Yan, Angew. Chem., Int. Ed., 2009, 48, 6499-6502.

23 J. R. Varcoe, Phys. Chem. Chem. Phys., 2007, 9, 1479-1486.

24 J. R. Varcoe, R. C. T. Slade, G. L. Wright and Y. L. Chen, J. Phys. Chem. B, 2006, 110, 21041-21049.

25 J. F. Zhou, M. Unlu, J. A. Vega and P. A. Kohl, J. Power Sources, 2009, 190, 285-292.

26 V. Lehman, Electrochemistry of Silicon, Wiley-VCH, Weinheim, Germany, 2002

27 B. M. Kayes, M. A. Filler, M. C. Putnam, M. D. Kelzenberg, N. S. Lewis and H. A. Atwater, Appl. Phys. Lett., 2007, 91, 103110.

28 R. B. Moore and C. R. Martin, Macromolecules, 1988, 21, 1334-1339.

29 S. Slade, S. A. Campbell, T. R. Ralph and F. C. Walsh, J. Electrochem. Soc., 2002, 149, A1556-A1564.

30 M. Inaba, T. Kinumoto, M. Kiriake, R. Umebayashi, A. Tasaka and Z. Ogumi, Electrochim. Acta, 2006, 51, 5746-5753.

31 A. Z. Weber and J. Newman, J. Electrochem. Soc., 2004, 151, A311A325.

32 M. D. Kelzenberg, S. W. Boettcher, J. A. Petykiewicz, D. B. TurnerEvans, M. C. Putnam, E. L. Warren, J. M. Spurgeon, R. M. Briggs, N. S. Lewis and H. A. Atwater, Nat. Mater., 2010, 9, 239-244.

33 G. C. Li and P. G. Pickup, Phys. Chem. Chem. Phys., 2000, 2, 1255 1260.

34 S. Sakamoto, M. Okumura, Z. G. Zhao and Y. Furukawa, Chem. Phys. Lett., 2005, 412, 395-398.

35 D. M. DeLongchamp and P. T. Hammond, Chem. Mater., 2003, 15, $1165-1173$.

36 D. DeLongchamp and P. T. Hammond, Adv. Mater., 2001, 13, $1455-$ 1459.

37 M. Unlu, J. F. Zhou and P. A. Kohl, J. Phys. Chem. C, 2009, 113, $11416-11423$.

38 M. Unlu, J. Zhou and P. A. Kohl, Fuel Cells, 2010, 10, 54-63. 